\title{
Variations on the adiabatic invariance: the Lorentz pendulum
}

\author{
Luis L. Sánchez-Soto and Jesús Zoido \\ Departamento de Óptica, Facultad de Física, Universidad Complutense, 28040 Madrid, Spain
}

(Dated: October 17, 2012)

\begin{abstract}
We analyze a very simple variant of the Lorentz pendulum, in which the length is varied exponentially, instead of uniformly, as it is assumed in the standard case. We establish quantitative criteria for the condition of adiabatic changes in both pendula and put in evidence their substantially different physical behavior with regard to adiabatic invariance.
\end{abstract}

\section{INTRODUCTION}

As early as 1902, Lord Rayleigh ${ }^{1}$ investigated a pendulum the length of which was being altered uniformly, but very slowly (some mathematical aspects of the problem were treated in 1895 by Le Cornd $\left.{ }^{2}\right)$. He showed that if $E(t)$ denotes the energy and $\ell(t)$ the length [or, equivalently, the frequency $v(t)]$ at a time $t$, then

$$
\frac{E(t)}{v(t)}=\frac{E(0)}{v(0)}
$$

This expression is probably the first explicit example of an adiabatic invariant; i.e., a conservation law that only holds when the parameters of the system are varied very slowly. The name was coined by analogy with thermodynamics, where adiabatic processes are those that occur sufficiently gently.

At the first Solvay Conference in 1911, Lorentz, unaware of Rayleigh's previous work, raised the question of the behavior of a "quantum pendulum" the length of which is gradually altered ${ }^{3}$ (by historical vicissitudes ${ }^{4}$ his name has become inextricably linked to that system). Einstein's reply was that "if the length of the pendulum is changed infinitely slowly, its energy remains equal to $h v$ if it is originally $h v$ ", although no detail of his analysis are given. In the same discussion, Warburg insisted that the length of the pendulum must be altered slowly, but not systematically. As Arnold aptly remarks5 "the person changing the parameters of the system must no see what state the system is in. Giving this definition a rigorous mathematical meaning is a very delicate and as yet unsolved problem. Fortunately, we can get along with a surrogate. The assumption of ignorance of the internal state of the system on the part of the person controlling the parameter may be replaced by the requirement that the change of parameter must be smooth; i.e., twice continuously differentiable". This important point is ignored in most expositions of adiabatic invariance.

Ehrenfest, who did not attend the Solvay Conference and was not cognizant of that discussion, had indeed read Rayleigh's paper and employed those ideas to enunciate his famous adiabatic principle ${ }^{6}$, which was promptly reformulated by Born and Fock ${ }^{7}$ in the form we now call adiabatic theorem ${ }^{8}$. In fact, this was a topic of uttermost importance in the old quantum theory910. To put it simply, if a physical quantity is going to make "all or nothing quantum jumps", it should make no jump at all if the system is perturbed gently, and therefore any quantized quantity should be an adiabatic invariant. The reader is referred to the book of Jammer ${ }^{111}$ for a masterful review of these questions, as well as the lucid and concise mathematical viewpoint of Levi-Civitta ${ }^{12 / 13}$.

The topic of adiabatic invariance has undergone a resurgence of interest from various different fields, such as plasma physics, thermonuclear research or geophysics 14 , although perhaps Berry's work on geometric phase ${ }^{15}$ has put it again in the spotlight. The two monographs by Sagdeev et a $t^{16}$ and Lochak and Meunie ${ }^{177}$ reflect this revival. More recently, the issue has renewed its importance in the context of quantum control (for example, concerning adiabatic passage between atomic energy level $\sqrt{18-23}$, , as well as adiabatic quantum computation $24 \mid 26$.

Adiabatic invariants are presented in most textbooks in terms of action-angle variables, which involves a significant level of sophistication. Even if a number of pedagogical papers has tried to alleviate these difficulties 27,34 , students often understand this notion only at a superficial level. Actually, perfunctory application of the adiabaticity condition may lead to controversial conclusions, even in the hands of experienced practitioners $35+38$.

Quite often the Lorentz pendulum is taken as a typical example to bring up this twist for graduate students39/40. In spite of its apparent simplicity, the proof of invariance is genuinely difficull ${ }^{41-48}$ and details are omitted. The purpose of this paper is to re-elaborate on this topic, putting forth pertinent physical discussion that emphasizes the motivation for doing what is done, as well as to present some variation of the Lorentz pendulum in the hope that its solution will shed light on the subject at an intermediate level.

\section{THE UNIFORMLY VARYING PENDULUM}

\section{A. Basic equations of motion}

We confine our attention to the ideal case of a simple pendulum of mass $m$ and variable length $\ell(t)$, oscillating under the gravity. The Lagrangian of the system is

$$
L=\frac{1}{2} m\left[\left(\frac{d \ell}{d t}\right)^{2}+\ell^{2}\left(\frac{d \vartheta}{d t}\right)^{2}\right]+m g \ell \cos \vartheta
$$

where $\vartheta(t)$ denotes the inclination of the pendulum with the vertical. The Euler-Lagrange equation for the generalized coordinate $\vartheta$ becomes

$$
\frac{d^{2} \vartheta}{d t^{2}}+\frac{2}{\ell}\left(\frac{d \ell}{d t}\right) \frac{d \vartheta}{d t}+\frac{g}{\ell} \sin \vartheta=0
$$


Note in passing that the length $\ell(t)$ acts as a geometrical (or holonomic) constraint, which here becomes time dependen 49950 .

In what follows we shall restrict ourselves to the regime of small oscillations (that is, $\sin \vartheta \simeq \vartheta$ ). In this Section, we deal with the example of a pendulum for which the length is uniformly altered in time; i. e.,

$$
\ell(t)=\ell_{0}(1+\varepsilon t),
$$

where $\varepsilon$ is a small parameter with the dimensions of a reciprocal time. It will be convenient to let $\tau=\varepsilon t$ and define a dimensionless time-dependent frequency

$$
\omega(\tau)=\frac{1}{\varepsilon} \sqrt{\frac{g}{\ell(\tau)}} .
$$

Equation 2.2. can be thus recast as

$$
\ddot{\vartheta}+2 \frac{\dot{\ell}}{\ell} \dot{\vartheta}+\omega^{2}(\tau) \vartheta=0
$$

where the dot represents differentiation with respect to $\tau$. We limit our analysis to a lengthening pendulum because if the amplitude of the initial displacement is small, then the resulting displacement will stay small. On the other hand, if the pendulum is shortening, then even if the initial displacement is small, the subsequent displacement will grow in time, violating the linearization hypothesis.

Using basic properties of Bessel functions ${ }^{51}$, the following

$$
\vartheta(\tau)=\frac{1}{\sqrt{1+\tau}}\left[A J_{1}\left(2 \omega_{0} \sqrt{1+\tau}\right)+B Y_{1}\left(2 \omega_{0} \sqrt{1+\tau}\right)\right],
$$

is a solution to (2.5), with $\omega_{0}=\omega(0)$ and $J_{n}(x)$ and $Y_{n}(x)$ the Bessel functions of $n$th order and first and second kind, respectively. The constants $A$ and $B$ must be determined by the initial conditions, which we take, without loss of generality, as $\vartheta(0)=\vartheta_{0}$ and $\dot{\vartheta}(0)=0$. The final result reads as

$$
\begin{aligned}
\vartheta(\tau) & =\frac{\pi \vartheta_{0} \omega_{0}}{\sqrt{1+\tau}}\left[J_{2}\left(2 \omega_{0}\right) Y_{1}\left(2 \omega_{0} \sqrt{1+\tau}\right)\right. \\
& \left.-Y_{2}\left(2 \omega_{0}\right) J_{1}\left(2 \omega_{0} \sqrt{1+\tau}\right)\right] .
\end{aligned}
$$

As a side comment, we remark that, in spite of its usual designation, neither Rayleigh nor Lorentz actually examined the behavior of this pendulum; this was first accomplished much later by Krutkov and Fock ${ }^{41}$, who obtained (2.7) and also derived Eq. (1.1) directly therefrom. Indeed, this solution allows one to investigate in great detail the periods of this system 39 .

Since $\varepsilon$ appears in the denominator in the definition (2.4), $\omega_{0}$ is actually very large (for example, if $\ell_{0}=1 \mathrm{~m}$ and $\varepsilon=$ $0.01 \mathrm{~s}^{-1}$, then $\omega_{0} \simeq 313$ ). This suggests to consider the limit

$$
2 \omega_{0} \sqrt{1+\tau} \gg 1
$$

and then take the leading term in the asymptotic expansion of the Bessel functions 51

$$
\begin{aligned}
& J_{n}(x) \sim \sqrt{\frac{2}{\pi x}} \cos \left(x-\frac{\pi}{4}-\frac{n \pi}{2}\right), \\
& Y_{n}(x) \sim \sqrt{\frac{2}{\pi x}} \sin \left(x-\frac{\pi}{4}-\frac{n \pi}{2}\right) .
\end{aligned}
$$

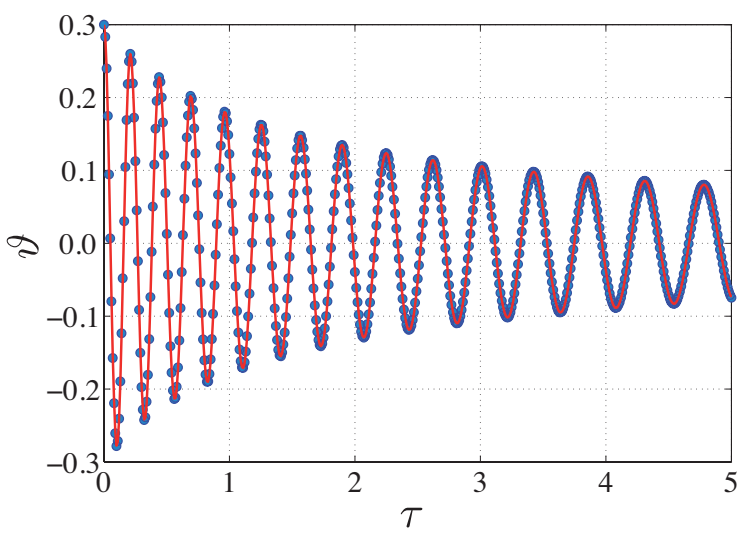

FIG. 1. Exact solution (red continuous line) and asymptotic approximation (blue points) for the uniformly varying pendulum with $\varepsilon=0.1 \mathrm{~s}^{-1}, \ell_{0}=1 \mathrm{~m}$ and $\vartheta_{0}=0.3 \mathrm{rad}$.

Consequently, we get

$$
\vartheta(\tau) \simeq \frac{\vartheta_{0}}{(1+\tau)^{3 / 4}} \cos \left[2 \omega_{0}(\sqrt{1+\tau}-1)\right] .
$$

By making use of the approximation 2.10, one can check that the maximum angular amplitude $\vartheta_{\text {max }}$ scales as

$$
\vartheta_{\max }(\tau)=\frac{\vartheta_{0}}{(1+\tau)^{3 / 4}}=\vartheta_{0}\left[\frac{\ell_{0}}{\ell(\tau)}\right]^{3 / 4},
$$

which shows that it is a decreasing function of time. Moreover, $\lim _{\tau \rightarrow \infty} \vartheta_{\max }(\tau)=0$.

In Fig. 1 both the exact solution 2.7 and its asymptotic approximation 2.10 are plotted. The error associated with 2.10 is very small; obviously, this error is smaller when $\tau$ is larger, since larger $\tau$ 's improve the approximation of 2.8. This can be formally expressed as

$$
\lim _{\tau \rightarrow \infty} 2 \omega_{0} \sqrt{1+\tau}=\infty
$$

This limit guarantees that, for a given value of $\omega_{0}$, the asymptotic expansion is always a good approximation for any $\tau$. From this perspective we can assert that the validity of the asymptotic expansion depends on the values of $\vartheta_{0}$ (and $\varepsilon$ ) but it is independent of time.

\section{B. Adiabatic invariance}

As pointed out in the Introduction, the concept of adiabatic change is associated with a variation that occurs infinitely slowly. The observer who is controlling the changes does not know the internal state of the system. In practical terms, this means that the change is adiabatic when the variation is carried out continuously and so slowly that the change $\delta \ell$ of the length is very small compared to the length $\ell$ of the pendu$\operatorname{lum} 52$; i.e.

$$
\frac{\delta \ell}{\ell} \ll 1
$$


By considering that the temporal interval in which the variation $\delta \ell$ is produced coincides with the local period $T$ for the length $\ell$, we have

$$
\delta \ell=T \frac{d \ell}{d t},
$$

and recalling that $T=2 \pi \sqrt{\ell / g}$, we can rewrite $\sqrt{2.13}$ for the example at hand as

$$
\frac{\delta \ell}{\ell}=\frac{\varepsilon T_{0}}{\sqrt{1+\tau}}=\frac{2 \pi}{\omega_{0} \sqrt{1+\tau}} \gg 1,
$$

with $T_{0}$ being the period of the pendulum at $\tau=0$. This requirement is independent of $\vartheta_{0}$ and, thus, independent of the amplitude of the oscillations, which is intuitively expected.

Equation (2.15) clearly suggests that if the change in length is initially adiabatic, it will remain forever. Moreover, the adiabatic character of the system will improve as time goes on.

Interestingly enough, condition $(2.15)$ is formally equivalent to 2.8 ensuring the validity of the asymptotic approximation; they will always be satisfied whenever

$$
\omega_{0} \gg \omega_{\lim }=2 \pi,
$$

which, from the definition of $\varepsilon$, can be equivalently recast as

$$
\varepsilon \ll \varepsilon_{\lim }=\frac{1}{2 \pi} \sqrt{\frac{g}{\ell_{0}}} .
$$

Equations 2.16) or 2.17) (which do not depend on time) provide a sensible criterion for the adiabatic change in a uniformly varying pendulum. The lesser the initial length, the more quickly can be lengthening the pendulum under the adiabatic hypothesis. Alternatively, $\omega_{\text {lim }}$ can be seen as the minimum value of $\omega_{0}$ for which the asymptotic expansion is valid independently of $\tau$.

To give a more quantitative argument, we compute the function

$$
I(\tau)=\frac{H(\tau)}{v(\tau)},
$$

that turns out to be an adiabatic invariant for arbitrary periodic motions in one degree of freedom 6 . Here $H(\tau)$ is the Hamiltonian and $v(\tau)$ the frequency of the oscillations.

For a pendulum, the Hamiltonian is

$$
H=\frac{1}{2} \frac{p^{2}}{\ell^{2}}+\frac{1}{2} g \ell \vartheta^{2},
$$

where $p=\ell^{2} d \vartheta / d t$ is the generalized momentum conjugate to $\vartheta$.

Taking into account the relations 51

$$
\frac{d}{d x}\left[x^{-1} J_{1}(x)\right]=x^{-1} J_{2}(x), \quad \frac{d}{d x}\left[x^{-1} Y_{1}(x)\right]=x^{-1} Y_{2}(x),
$$

and the solution 2.7), the angular velocity of the pendulum can be expressed as

$$
\dot{\vartheta}=\frac{\pi \vartheta_{0} \omega_{0} \varepsilon}{1+\tau} \mathscr{H}_{22}\left(2 \omega_{0} \sqrt{1+\tau}\right)
$$

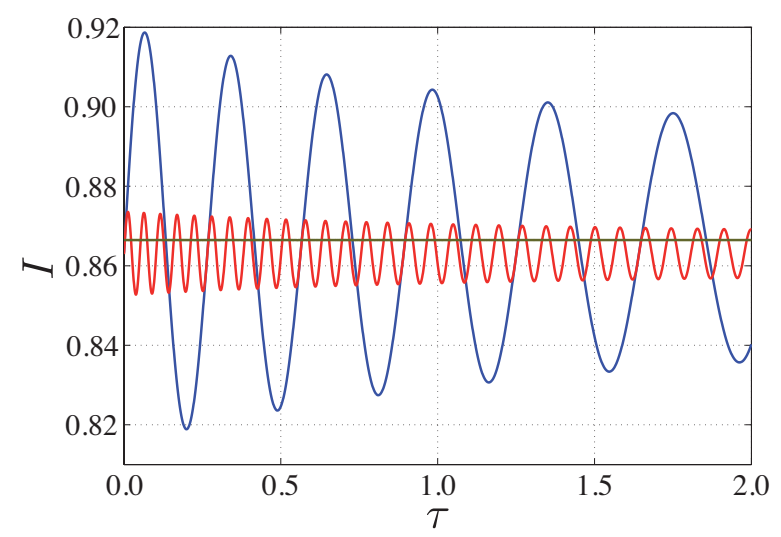

FIG. 2. Plot of $I(\tau)$ as a function of $\tau$ for the uniformly varying pendulum with $\ell_{0}=1 \mathrm{~m}$ and $\vartheta_{0}=0.3 \mathrm{rad}$. The curves corresponds to $\omega_{0}=2,10$ and 1000 (which are associated with the values $\varepsilon=0.2491,0.0498$ and $\left.0.00005 \mathrm{~s}^{-1}\right)$, following the decreasing amplitudes.

which immediately leads to

$$
H(\tau)=H_{0} \pi^{2} \omega_{0}^{2}\left[\mathscr{H}_{22}^{2}\left(2 \omega_{0} \sqrt{1+\tau}\right)+\mathscr{H}_{21}^{2}\left(2 \omega_{0} \sqrt{1+\tau}\right)\right] .
$$

Here, for notational simplicity, we have introduced the functions

$$
\begin{aligned}
& \mathscr{H}_{21}(x)=J_{2}\left(2 \omega_{0}\right) Y_{1}(x)-Y_{2}\left(2 \omega_{0}\right) J_{1}(x), \\
& \mathscr{H}_{22}(x)=Y_{2}\left(2 \omega_{0}\right) J_{2}(x)-J_{2}\left(2 \omega_{0}\right) Y_{2}(x),
\end{aligned}
$$

and $H_{0}=\vartheta_{0} \omega_{0}^{2} \varepsilon^{2} \ell_{0}^{2} / 2$ is the total energy at $\tau=0$. Finally, since $v(\tau)=v_{0} / \sqrt{1+\tau}$, with $v_{0}=\omega_{0} \varepsilon / 2 \pi$, we get

$$
\begin{aligned}
I(\tau) & =\frac{H_{0}}{v_{0}} \sqrt{1+\tau} \pi^{2} \omega_{0}^{2} \\
& \times\left[\mathscr{H}_{22}^{2}\left(2 \omega_{0} \sqrt{1+\tau}\right)+\mathscr{H}_{21}^{2}\left(2 \omega_{0} \sqrt{1+\tau}\right)\right] .
\end{aligned}
$$

Because $I(\tau)$ is a time-dependent function, it will not be, in general, an adiabatic invariant. In other words, for arbitrary values of the parameters, the solution 2.77 will not be associated with adiabatic changes in the length of the pendulum. The function $I(\tau)$ is shown in Fig. 2 for different values of $\omega_{0}$. One immediately concludes that the larger $\omega_{0}$, the lesser time-dependent $I(\tau)$ becomes, which is in full agreement with (2.16).

To complete the analysis, we proceed to calculate $I(\tau)$ with the asymptotic approximations for the Bessel functions as in Eq. 2.10). After a direct manipulation, we end up with

$$
H(\tau) \simeq \frac{H_{0}}{\sqrt{1+\tau}}
$$

so that

$$
I(\tau) \simeq \frac{H_{0}}{v_{0}}=\pi \ell_{0}^{3 / 2} \vartheta_{0}^{2} g^{1 / 2} .
$$

This is an important result: for large enough values of $\tau$, the quantity $I(\tau)$ becomes an adiabatic invariant. This validates 
the previously suggested conclusion: the condition establishing the validity of the asymptotic expansion of the Bessel functions is conceptually equivalent to the condition of adiabatic invariance.

\section{THE EXPONENTIALLY VARYING PENDULUM}

\section{A. Basic equations of motion}

We turn now our attention to the instance where the length of the pendulum is altered non uniformly. More concretely, we take

$$
\ell(t)=\ell_{0} e^{\varepsilon t}
$$

Equation 2.2, when small oscillations are considered, reduces in this case to

$$
\ddot{\vartheta}+2 \dot{\vartheta}+\omega_{0}^{2} e^{-\tau} \vartheta=0
$$

The change $\vartheta=\theta e^{-\tau}$ gives

$$
\ddot{\theta}+\left(\omega_{0}^{2} e^{-\tau}-1\right) \theta=0
$$

which has again an exact solution in terms of Bessel functions 53 . The result, employing the original variables, reads as

$$
\vartheta(\tau)=e^{-\tau}\left[A J_{2}\left(2 \omega_{0} e^{-\tau / 2}\right)+B Y_{2}\left(2 \omega_{0} e^{-\tau / 2}\right)\right] .
$$

To fix the constants $A$ and $B$ we take the same initial conditions as before, namely $\vartheta(0)=\vartheta_{0}$ and $\dot{\vartheta}(0)=0$. Applying the relations

$$
\frac{d}{d x}\left[x^{2} J_{2}(x)\right]=x^{2} J_{1}(x), \quad \frac{d}{d x}\left[x^{2} Y_{2}(x)\right]=x^{2} Y_{1}(x)
$$

in conjunction with the change of variable $x=2 \omega_{0} e^{-\tau / 2}$ and the Wronskian

$$
J_{1}(x) Y_{2}(x)-J_{2}(x) Y_{1}(x)=-\frac{2}{\pi x},
$$

one can show that the final solution is

$$
\begin{aligned}
\vartheta(\tau) & =\pi \vartheta_{0} \omega_{0} e^{-\tau}\left[Y_{1}\left(2 \omega_{0}\right) J_{2}\left(2 \omega_{0} e^{-\tau / 2}\right)\right. \\
& \left.-J_{1}\left(2 \omega_{0}\right) Y_{2}\left(2 \omega_{0} e^{-\tau / 2}\right)\right] .
\end{aligned}
$$

Much in the same way as for the pendulum with uniformly varying length, if

$$
2 \omega_{0} e^{-\tau / 2} \gg 1
$$

is satisfied, we can replace equation 3.7 by its asymptotic approximation, leading to

$$
\vartheta(\tau) \simeq \vartheta_{0} e^{-3 \tau / 4} \cos \left[2 \omega_{0}\left(1-e^{-\tau / 2}\right)\right]
$$

Within this approximation the maximum angular amplitude $\vartheta_{\max }$ scales exactly as in Eq. 2.11, and also $\lim _{\tau \rightarrow \infty} \vartheta_{\max }(\tau)=0$. However, as expected, the angular amplitude $\vartheta_{\text {max }}$ falls off more quickly in this case. This behavior

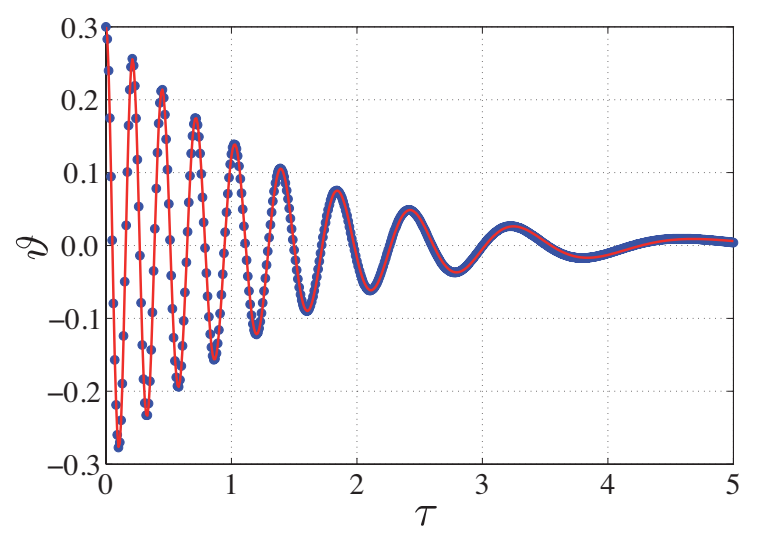

FIG. 3. Plot of the exact solution (in red) and the asymptotic approximation (blue points) for the exponentially varying pendulum with the same parameters as in Fig. 1.

can be clearly appreciated in Fig. 3, where both the exact solution (3.7) and its asymptotic approximation (3.9) are plotted. The agreement is again remarkable.

In contradistinction with the situation described by Eq. 2.12, the exponentially varying pendulum leads to the limit relation

$$
\lim _{\tau \rightarrow \infty} 2 \omega_{0} e^{-\tau / 2}=0
$$

This points out the more important conceptual difference between these two cases: for the uniformly varying pendulum the validity of the asymptotic expansion of the Bessel functions only depends on the values of $\varepsilon$ and $\vartheta_{0}$, but it is independent on the time. For the exponentially varying pendulum the validity of that approximation is time dependent and for large values of the time this approximation breaks down.

At first glance, this important difference between the two penduli can seems only a formal one. However, as we shall see in the following it has strong implications for the adiabatic invariance.

\section{B. Adiabatic invariance}

We next analyze the adiabatic change for this example. Equation 2.14 applied to 3.1 gives as a requirement for adiabatic invariance

$$
\frac{\delta \ell}{\ell}=\varepsilon T_{0} e^{\tau / 2}=\frac{2 \pi}{\omega_{0}} e^{\tau / 2} \ll 1,
$$

which again is formally equivalent to the condition 3.8 for the validity of the asymptotic approximation of the Bessel functions.

This indicates that even if the change of length is initially adiabatic, it will remain so only for a finite interval of time. To put in another way, (3.11) holds true whenever

$$
\omega_{0} \gg e^{\tau / 2} \omega_{\lim }=2 \pi e^{\tau / 2}
$$




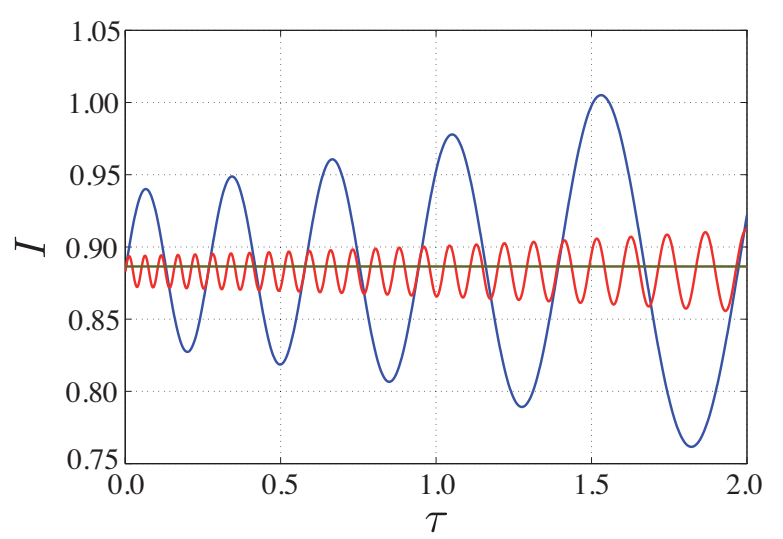

FIG. 4. Plot of $I(\tau)$ as a function of $\tau$ for the exponentially varying pendulum with $\ell_{0}=1 \mathrm{~m}$ and $\vartheta_{0}=0.3 \mathrm{rad}$. The curves corresponds to $\omega_{0}=2,10$ and 1000 (which are associated with the values $\varepsilon=0.2491,0.0498$ and $\left.0.00005 \mathrm{~s}^{-1}\right)$, following the decreasing amplitudes.

or, recalling the definition of $\omega(\tau)$,

$$
\tau \ll \tau_{\lim }=2 \ln \left(\frac{1}{\varepsilon T_{0}}\right) .
$$

Accordingly, there does not exist a minimum fixed value of $\omega_{0}$ such that adiabaticity holds true forever. In our opinion this is the more important lesson from this paper: the adiabatic condition does not need to hold, in general, for all times.

The formal equivalence discussed so far provides a mathematical interpretation fo (3.13): the change will be adiabatic in those conditions in which the asymptotic expansion of Bessel functions is justified.

Note that the change of the length in this example is infinitely continuously differentiable, but the adiabaticity holds true only in a time interval fixed by the values of $\ell_{0}$ and $\varepsilon$. This by no means contradict Arnold's adiabaticity requirement mentioned in the Introduction (that is, that the change of the length must be twice continuously differentiable), since Arnold explicitly considers a finite time interval in the definition of the adiabatic invariants ${ }^{5}$.

Finally, we calculate explicitly the total energy for this case. Using again the Hamiltonian (2.19) and the solution (3.7) and its time derivative, we get

$$
\begin{aligned}
H(\tau) & =H_{0} \pi^{2} \omega_{0}^{2} e^{-\tau} \\
& \times\left[\mathscr{H}_{11}^{2}\left(2 \omega_{0} e^{-\tau / 2}\right)+\mathscr{H}_{12}^{2}\left(2 \omega_{0} e^{-\tau / 2}\right)\right],
\end{aligned}
$$

with

$$
\begin{aligned}
& \mathscr{H}_{12}(x)=Y_{1}\left(2 \omega_{0}\right) J_{2}(x)-J_{1}\left(2 \omega_{0}\right) Y_{2}(x), \\
& \mathscr{H}_{11}(x)=J_{1}\left(2 \omega_{0}\right) Y_{1}(x)-Y_{1}\left(2 \omega_{0}\right) J_{1}(x) .
\end{aligned}
$$

In consequence, $I(\tau)$ becomes

$$
I(\tau)=\frac{H_{0}}{v_{0}} \pi^{2} \omega_{0}^{2} e^{-\tau / 2}\left[\mathscr{H}_{11}^{2}\left(2 \omega_{0} e^{-\tau / 2}\right)+\mathscr{H}_{12}^{2}\left(2 \omega_{0} e^{-\tau / 2}\right)\right] .
$$

The function $I(\tau)$ is represented in Fig. 4. As we can see, the fluctuations of $I(\tau)$ increase with time. Thus, for large enough time, $I(\tau)$ will never be an invariant quantity, irrespective of the value of $\omega_{0}$. However, for the time window chosen in the figure, we see that for $\omega_{0}=1000, I(\tau)$ looks invariant over the entire interval.

Our last step is to calculate $I(\tau)$ using the asymptotic approximations for the Bessel functions. Now, we have

$$
H(\tau) \simeq H_{0} e^{-\tau / 2}
$$

so that

$$
I(\tau) \simeq \frac{H_{0}}{v_{0}}=\pi \ell_{0}^{3 / 2} \vartheta_{0}^{2} g^{1 / 2},
$$

which is identical to what we have obtained for the uniformly varying pendulum.

\section{CONCLUDING REMARKS}

We have explored in detail two nontrivial yet solvable examples of penduli of varying length with the purpose of a better understanding of the concept of adiabatic invariance.

The ambiguous criteria of "infinitely slow variation", or the assumption of "ignorance of the internal state of the system on the part of the person controlling the variable parameter", usually employed to establish the condition of adiabatic change, are replaced here by more quantitative criteria.

For the two penduli considered in this paper, we have shown that the physical meaning of adiabatic change is formally contained in the mathematical condition of validity for the asymptotic expansion of the Bessel functions: the validity of the asymptotic approximation implies adiabatic change and vice versa.

The analysis carried out in this work invites to a more general reflection: it is important to pay special attention to the meaning of mathematical approximations. Actually, the radius of convergence of some systematic approximation to an exact solution has always a physical origin.

\section{ACKNOWLEDGMENTS}

The original ideas in this paper originated from a long cooperation with the late Richard Barakat. Over the years, they have been further developed and completed with questions, suggestions, criticism, and advice from many students and colleagues. Particular thanks for help in various ways go to E. Bernabéu, J. F. Cariñena, A. Galindo, H. de Guise, H. Kastrup, A. B. Klimov, G. Leuchs and J. J. Monzón.

We are indebted to two anonymous referees for valuable comments.

This paper is dedicated to the memory of coauthor J. Zoido, who unexpectedly passed away during the preparation of the final version.

This work is partially supported by the Spanish DGI (Grants FIS2008-04356 and FIS2011-26786) and the UCMBSCH program (Grant GR-920992). 
1 J. W. S. Rayleigh, "On the pressure of vibrations," Phil. Mag. 3, 338-346 (1902).

2 L. Le Cornu, "Mémoire sur le pendule de longueur variable," Acta Math. 19, 201-249 (1895).

3 P. Langevin and M. D. Broglie, eds., La Theorie du Rayonnement et les Quanta (Gauthier-Villars,, Paris, 1912).

4 L. Navarro and E. Pérez, "Paul Ehrenfest: The genesis of the adiabatic hypothesis, 1911-1914," Arch. Hist. Exact Sci. 60, 209-267 (2006).

5 V. I. Arnold, Mathematical Methods of Classical Mechanics (Springer, 1978).

6 P. Ehrenfest, "Adiabatische Invarianten und Quantentheorie," Ann. Phys. (Berlin) 51, 327-352 (1916).

7 M. Born and V. Fock, "Beweis des Adiabatensatzes," Z. Phys. 51, 165-180 (1928).

8 T. Kato, "On the adiabatic theorem of quantum mechanics," J. Phys. Soc. Jpn. 5, 435-439 (1950).

9 A. Sommerfeld, Atombau und Specktrallinien (Vieweg, Braunschweig, 1919).

10 M. Born, Vorlesungen über Atommechanik (Springer, Berlin, 1925).

11 M. Jammer, The Conceptual Development of Quantum Mechanics (McGraw-Hill, 1966).

12 T. Levi-Civita, "Drei Vorlesungen über adiabatische Invarianten," Abh. Math. Sem. Hamburg 6, 323-366 (1928).

13 T. Levi-Civita, "A general survey of the theory of adiabatic invariants," J. Math. Phys. Camb. 13, 18-40 (1934).

14 K. J. Whiteman, "Invariants and stability in classical mechanics," Rep. Prog. Phys. 40, 1033-1069 (1977).

15 A. Shapere and F. Wilczek, eds., Geometric Phases in Physics (World Scientific, Singapore, 1989).

16 R. Z. Sagdeev, D. A. Usikov, and G. M. Zaslavsky, Nonlinear Physics: From The Pendulum To Turbulence And Chaos (Harwood, New York, 1988).

17 P. Lochak and C. Meunier, Multiphase Averaging for Classical Systems. With Applications to Adiabatic Theorems (Springer, New York, 1988).

18 J. Oreg, F. T. Hioe, and J. H. Eberly, "Adiabatic following in multilevel systems," Phys. Rev. A 29, 690-697 (1984).

19 U. Gaubatz, P. Rudecki, M. Becker, S. Schiemann, M. Külz, and K. Bergmann, "Population switching between vibrational levels in molecular beams," Chem. Phys. Lett. 149, 463-468 (1988).

20 U. Gaubatz, P. Rudecki, S. Schiemann, and K. Bergmann, "Population transfer between molecular vibrational levels by stimulated Raman scattering with partially overlapping laser fields. A new concept and experimental results," J. Chem. Phys. 92, 5363-5376 (1990).

21 S. Schiemann, A. Kuhn, S. Steuerwald, and K. Bergmann, "Efficient coherent population transfer in NO molecules using pulsed lasers," Phys. Rev. Lett. 71, 3637-3640 (1993).

22 P. Pillet, C. Valentin, R. L. Yuan, and J. Yu, "Adiabatic population transfer in a multilevel system," Phys. Rev. A 48, 845-848 (1993).

23 P. Král, I. Thanopulos, and M. Shapiro, "Colloquium: Coherently controlled adiabatic passage,” Rev. Mod. Phys. 79, 53-77 (2007).

24 E. Farhi, J. Goldstone, S. Gutmann, and M. Sipser, "Quantum computation by adiabatic evolution,"

25 E. Farhi, J. Goldstone, S. Gutmann, J. Lapan, A. Lundgren, and D. Preda, "A quantum adiabatic evolution algorithm applied to random instances of an np-complete problem," Science 292, 472475 (2001).

26 J. Pachos and P. Zanardi, "Quantum holonomies for quantum computing,” Int. J. Mod. Phys. B 15, 1257-1286 (2001).

27 L. Parker, "Adiabatic invariance in simple harmonic motion," Am. J. Phys. 39, 24-27 (1971).

28 M. G. Calkin, "Adiabatic invariants for varying mass," Am. J. Phys. 45, 301-302 (1977).

29 C. Gignoux and F. Brut, "Adiabatic invariance or scaling?" Am. J. Phys. 57, 422-428 (1989).

30 F. S. Crawford, "Elementary examples of adiabatic invariance," Am. J. Phys. 58, 337-344 (1990).

31 J. L. Anderson, "Multiple time scale methods for adiabatic systems," Am. J. Phys. 60,, 923-927 (1992).

32 A. C. Aguiar Pinto, M. C. Nemes, J. G. Peixoto de Faria, and M. T. Thomaz, "Comment on the adiabatic condition," Am. J. Phys. 68, 955-958 (2000).

33 C. G. Wells and S. T. C. Siklos, "The adiabatic invariance of the action variable in classical dynamics," Eur. J. Phys 28, 105-112 (2007).

34 B. W. Shore, M. V. Gromovyy, L. P. Yatsenko, and V. I. Romanenko, "Simple mechanical analogs of rapid adiabatic passage in atomic physics," Am. J. Phys. 77, 1183-1194 (2009).

35 K.-P. Marzlin and B. C. Sanders, "Inconsistency in the application of the adiabatic theorem," Phys. Rev. Lett. 93, 160408 (2004).

36 M. S. Sarandy, L.-A. Wu, and D. Lidar, "Consistency of the adiabatic theorem," Quantum Inf. Process. 3, 331-349 (2004).

37 J. Du, L. Hu, Y. Wang, J. Wu, M. Zhao, and D. Suter, "Experimental study of the validity of quantitative conditions in the quantum adiabatic theorem," Phys. Rev. Lett. 101, 060403 (2008).

38 M. H. S. Amin, "Consistency of the adiabatic theorem," Phys. Rev. Lett. 102, 220401 (2009).

39 T. Wickramasinghe and R. Ochoa, "Analysis of the linearity of half periods of the Lorentz pendulum," Am. J. Phys. 73, 442-445 (2005).

40 A. Kavanaugh and T. Moe, "The pit and the pendulum," http://online.redwoods.cc.ca.us/instruct/darnold/deproj/sp05/atrav/ThePitandTl (2005).

41 G. Krutkow and V. Fock, "Über das Rayleighesche Pendel," Z. Phys. 13, 195-202 (1923).

42 R. M. Kulsrud, "Adiabatic invariant of the harmonic oscillator," Phys. Rev. 106, 205-207 (1957).

43 C. S. Gardner, "Adiabatic invariants of periodic classical systems," Phys. Rev. 115, 791-794 (1959).

44 M. Kruskal, "Asymptotic theory of Hamiltonian and other systems with all solutions nearly periodic," J. Math. Phys. 3, 806828 (1962).

45 J. E. Littlewood, "Lorentz's pendulum problem," Ann. Phys. 21, 233-242 (1963).

46 M. N. Brearley, "The simple pendulum with uniformly changing string length,” Proc. Edin. Math. Soc. 15, 61-66 (1966).

47 A. Werner and C. J. Eliezer, "The lengthening pendulum,” J. Aust. Math. Soc. 9, 331-336 (1969).

48 D. K. Ross, "The behaviour of a simple pendulum with uniformly shortening string length,” Int. J. Nonlin. Mech. 14, 175182 (1979).

49 H. Goldstein, Classical Mechanics (Addison-Wesley, New York, 1980).

50 J. V. José and E. J. Saletan, Classical Dynamics: A Contemporary Approach (Cambridge University Press, Cambridge, 1998).

51 N. W. McLachlan, Bessel Functions for Engineers (Oxford University Press, Oxford, 1955).

52 C. Andrade, The Structure of the Atom (Harcourt, New York, 1962). 
53 E. Kamke, Differentialgleichungen: Lösungsmethoden und

Lösungen, vol. 1 (Chelsea, New York, 1974). 